UDC: 519.658 .4

\title{
Extension of Strongin's Global Optimization Algorithm to a Function Continuous on a Compact Interval
}

\author{
V. I. Zabotin ${ }^{\mathrm{a}}$, P. A. Chernyshevskij ${ }^{\mathrm{b}}$ \\ Tupolev Kazan National Research Technical University - KAI, \\ Academic Building No. 7, 55 Bolshaya Krasnaya Str., Kazan, Republic of Tatarstan, Russia \\ E-mail: ${ }^{a}$ v.zabotin@rambler.ru, ${ }^{\mathrm{b}}$ pavelcommer@gmail.com
}

Received 26.05.2019.

Accepted for publication 14.11.2019.

\begin{abstract}
The Lipschitz continuous property has been used for a long time to solve the global optimization problem and continues to be used. Here we can mention the work of Piyavskii, Yevtushenko, Strongin, Shubert, Sergeyev, Kvasov and others. Most papers assume a priori knowledge of the Lipschitz constant, but the derivation of this constant is a separate problem. Further still, we must prove that an objective function is really Lipschitz, and it is a complicated problem too. In the case where the Lipschitz continuity is established, Strongin proposed an algorithm for global optimization of a satisfying Lipschitz condition on a compact interval function without any a priori knowledge of the Lipschitz estimate. The algorithm not only finds a global extremum, but it determines the Lipschitz estimate too. It is known that every function that satisfies the Lipchitz condition on a compact convex set is uniformly continuous, but the reverse is not always true. However, there exist models (Arutyunova, Dulliev, Zabotin) whose study requires a minimization of the continuous but definitely not Lipschitz function. One of the algorithms for solving such a problem was proposed by R. J. Vanderbei. In his work he introduced some generalization of the Lipchitz property named $\varepsilon$-Lipchitz and proved that a function defined on a compact convex set is uniformly continuous if and only if it satisfies the $\varepsilon$-Lipchitz condition. The above-mentioned property allowed him to extend Piyavskii's method. However, Vanderbei assumed that for a given value of $\varepsilon$ it is possible to obtain an associate Lipschitz $\varepsilon$-constant, which is a very difficult problem. Thus, there is a need to construct, for a function continuous on a compact convex domain, a global optimization algorithm which works in some way like Strongin's algorithm, i.e., without any a priori knowledge of the Lipschitz $\varepsilon$-constant. In this paper we propose an extension of Strongin's global optimization algorithm to a function continuous on a compact interval using the $\varepsilon$-Lipchitz conception, prove its convergence and solve some numerical examples using the software that implements the developed method.
\end{abstract}

Keywords: $\varepsilon$-Lipschitz functions, function minimization, Strongin's algorithm, algorithm convergence

Citation: Computer Research and Modeling, 2019, vol. 11, no. 6, pp. 1111-1119.

(C) 2019 Vladislav I. Zabotin, Pavel A. Chernyshevskij This work is licensed under the Creative Commons Attribution-NoDerivs 3.0 Unported License. To view a copy of this license, visit http://creativecommons.org/licenses/by-nd/3.0/ or send a letter to Creative Commons, PO Box 1866, Mountain View, CA 94042, USA. 


\section{Introduction}

The Lipschitz continuous property is widely used in theoretical research and in solving applied problems. In particular, being a global property of a function, the Lipschitz continuity can be used to solve the global optimization problem.

The first studies in which methods for optimizing a Lipschitz continuous function were proposed are those of S. A. Piyavskii [Piyavskij, 1967; Piyavskij, 1972], Yu. G. Yevtushenko [Yevtushenko, 1971], B. Shubert [Shubert, 1972], and R. G. Strongin [Strongin, 1978]. To date the amount of research on this topic has increased significantly. Of special note here is the book of Ya. D. Sergeyev and D. E. Kvasov [Sergeyev, Kvasov, 2008] (see also references therein).

The extension of the class of optimizable functions is of interest. One of the possibilities for such an extension is proposed in R. J. Vanderbei's paper [Vanderbei, 1999], where the idea of $\varepsilon$-Lipschitz functions was represented: a function $f(x)$ defined on a convex domain $A \subset \mathbb{R}^{n}$ is $\varepsilon$-Lipschitz if condition (1) holds:

$$
\forall \varepsilon>0 \exists L(\varepsilon)<+\infty \forall x, y \in A:|f(x)-f(y)| \leq L(\varepsilon)\|x-y\|+\varepsilon .
$$

In the same work the following theorem was proved: if $A$ is a convex compact domain, then the continuity of the function on $A$ and the $\varepsilon$-Lipschitz continuity on $A$ are equivalent notions.

Note that the idea of $\varepsilon$-Lipschitz functions is not a trivial extension of the Lipschitz functions because not every continuous function is Lipschitz, for example, $f(x)=\sqrt{|x|}$ on $(-1 ; 1)$. Moreover, it is well known that a Lipschitz function is almost everywhere differentiable and there are known examples (in particular, the Weierstrass function [Gelbaum, Olmsted, 2003, p. 39]) of a nowhere differentiable continuous functions.

Finally, if there are some instruments for estimating the Lipschitz constant, for example, a function continuous on a compact $A$ gradient, then in the case of $\varepsilon$-Lipschitz functions we have no such universal analytic tools of finding $L(\varepsilon)$ for a given $\varepsilon>0$. Finding $L(\varepsilon)$ as a function of $\varepsilon>0$ involves enough difficulties as can be seen from the work [Vanderbei, 1999] where an explicit form of dependence of $L(\varepsilon)$ on $\varepsilon>0$ was obtained for functions $f(x)=x^{\alpha}, x \in[0 ; b], 0<\alpha<1$ and $f(x)=\frac{1}{\ln x}, x \in(0 ; a]$; from the work [Arutyunova, 2013] where similar dependences were established for the function $f(x)=\min \left\{\sqrt{\left|x-a_{1}\right|}+b_{1}, \sqrt{\left|x-a_{2}\right|}+b_{2}, \sqrt{\left|x-a_{3}\right|}+b_{3}\right\}$; and from the work [Arutyunova et al., 2017] in which an estimate of $L(\varepsilon)$ was found for $f(x)=\arcsin x$ defined on $[-1 ; 1]$.

One of the important applications of the $\varepsilon$-Lipschitzian functions is their optimization. Such problems appear as an auxiliary task in constructing various numerical algorithms for projection of a point onto a set of a different nature, for example, in [Arutyunova et al., 2017; Zabotin, Arutyunova, 2013; Arutyunova et al., 2014; Chernyaev, 2016; Zabotin, Chernyaev, 2016; Chernyaev, 2015] and are of interest in their own right: in [Vanderbei, 1999] Piyavskii's algorithm was extended to $\varepsilon$-Lipschitzian functions and in [Arutyunova, 2013] the modification of Yevtushenko's method for the $\varepsilon$-Lipschitz functions defined on a compact interval was proposed.

However, all works listed above generally assume a priori knowledge of the dependence of a constant $L(\varepsilon)$ on $\varepsilon>0$, but this requires solving the complicated problem as noted above.

Thus, there is a need to create a numerical algorithm for an approximate evaluation of a minimal value of the constant $L(\varepsilon)$ for a given value of $\varepsilon>0$. One of the possible methods for solving this problem is obtained in [Zabotin, Chernyshevskij, 2018].

In this paper, we consider a numerical minimization algorithm for a function continuous on a compact interval, and this algorithm does not require a priori knowledge of the $L(\varepsilon)$ estimate and extends, in some ways, Strongin's algorithm [Strongin, 1978; Vasil'ev, 1988]. 


\section{Description of the algorithm and its proof}

Note that the definition (1) does not guarantee a positive value of $L(\varepsilon)$. To avoid negative values of the $L(\varepsilon)$ minimal estimate (which is attained as shown below), we can use a definition from [Zabotin, Chernyshevskij, 2018]: a function $f$ and a number $\varepsilon>0$ are conformed on a set $A$ if there exists a $x_{0}, y_{0} \in A$ such that $0<\varepsilon<\left|f\left(x_{0}\right)-f\left(y_{0}\right)\right|$. In [Arutyunova et al., 2014] the value $l(\varepsilon)=\inf \{L(\varepsilon)\}$ is introduced, $\{L(\varepsilon)\}$ being the set of $L(\varepsilon)$ estimates for a fixed $\varepsilon>0$. It follows from Proposition 1 in [Zabotin, Chernyshevskij, 2018] that $l(\varepsilon) \in\{L(\varepsilon)\}$ and $l(\varepsilon)>0$ for a function $f$ conformed on $A$ and $\varepsilon>0$.

Throughout this paper we suppose that the conformity constraint is met and it is obvious that this condition can be easily checked.

\section{Algorithm A.}

In:

1) a function $f(x)$ continuous on a compact interval $[a ; b]$;

2) a value $\varepsilon>0$ and the function $f(x)$ conformed on $[a ; b]$.

\section{Out:}

1) an approximate value of $f_{*}=\min _{x \in[a ; b]} f(x)$;

2) a point $x_{*}$ from $[a ; b]$ in which such an approximate value is attained;

3 ) a lower estimate of the minimal Lipschitz constant $l(\varepsilon)$ determined for the function $f$ on $[a ; b]$.

Step 0. Pick two points $u_{0}=v_{0}=a$ and $u_{1}=v_{1}=b$. Set method parameters $\nu>0, \mu_{0}>1$ and a bounded sequence $\mu_{k}>1, k=1,2, \ldots$ (which is a method parameter too). Put $k=1$.

Step 1. Calculate the value $L_{k}(\varepsilon)$ :

$$
L_{k}(\varepsilon)= \begin{cases}\mu_{k} l_{k}(\varepsilon), & l_{k}(\varepsilon)>0 \\ \nu, & l_{k}(\varepsilon) \leq 0\end{cases}
$$

where

$$
l_{k}(\varepsilon)=\max _{0 \leq j<i \leq k} \frac{\left|f\left(u_{i}\right)-f\left(u_{j}\right)\right|-\varepsilon}{u_{i}-u_{j}} .
$$

Step 2. For every interval $\left[u_{i-1} ; u_{i}\right]$ find the characteristic

$$
R_{k}(i, \varepsilon)=L_{k}(\varepsilon)\left(u_{i}-u_{i-1}\right)+\frac{\left(f\left(u_{i}\right)-f\left(u_{i-1}\right)\right)^{2}}{L_{k}(\varepsilon)\left(u_{i}-u_{i-1}\right)+\varepsilon}-2\left(f\left(u_{i}\right)+f\left(u_{i-1}\right)\right), i=1, \ldots, k,
$$

which plays the same role as the value $R(i)$ proposed by Strongin in [Strongin, 1978, p. 79].

Then determine the minimal number $s$ such that:

$$
R_{k}(s, \varepsilon)=\max _{1 \leq i \leq k} R_{k}(i, \varepsilon), \quad R_{k}(i, \varepsilon)<R_{k}(s, \varepsilon), \quad i=1, \ldots, s-1 .
$$

Step 3. Put $L_{k}{ }^{\prime}(\varepsilon)=L_{k}(\varepsilon)$.

Step 4. Calculate

$$
v_{k+1}=\frac{\left(u_{s}+u_{s-1}\right)}{2}-\frac{\left(f\left(u_{s}\right)-f\left(u_{s-1}\right)\right)}{2 L_{k}^{\prime}(\varepsilon)}
$$

and check the constraint

$$
u_{s-1}<v_{k+1}<u_{s} .
$$

If condition (7) is not satisfied, then put $L_{k}{ }^{\prime}(\varepsilon)=\mu_{0} L_{k}{ }^{\prime}(\varepsilon)$ and return to step 4. If the constraint is satisfied, then go to step 5 .

Step 5. Check the termination condition (which can be selected arbitrarily). If it is satisfied, then put $f_{*}=f\left(v_{k+1}\right), x_{*}=v_{k+1}, l(\varepsilon)=l_{k}(\varepsilon)$ and stop. If the condition is not satisfied, then reindex points $u_{0}, \ldots, u_{k}, v_{k+1}$ in the order of increasing such that $u_{0}=a<u_{1}<\ldots<u_{k}<u_{k+1}=b$, put $k=k+1$ and return to step 1 . The algorithm is formulated. 


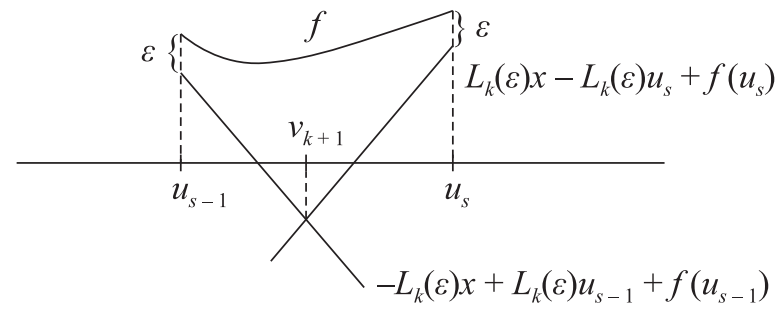

Figure 1. A situation where the (next) point $v_{k+1}$ obtained by the proposed search algorithm satisfies the condition $u_{s-1}<v_{k+1}<u_{s}$, i.e., it is inside the current search interval $\left[u_{s-1}, u_{s}\right]$. This situation is guaranteed for the Lipschitz functions

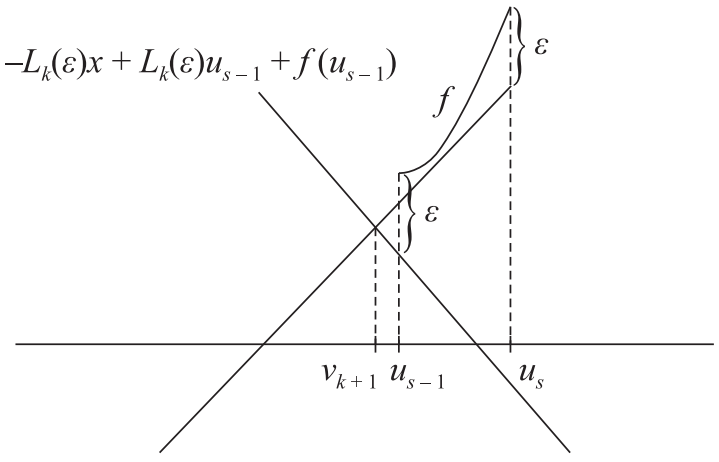

Figure 2. A situation where the (next) point $v_{k+1}$ obtained by the proposed search algorithm does not satisfy the condition $u_{s-1}<v_{k+1}<u_{s}$, i.e., it is outside the current search interval $\left[u_{s-1}, u_{s}\right]$. This situation is possible because of the $\varepsilon$-Lipschitz condition, but it cannot happen in the case of Lipschitz functions. It is easy to see from the figure that the inequality $v_{k+1}<u_{s-1}$ holds

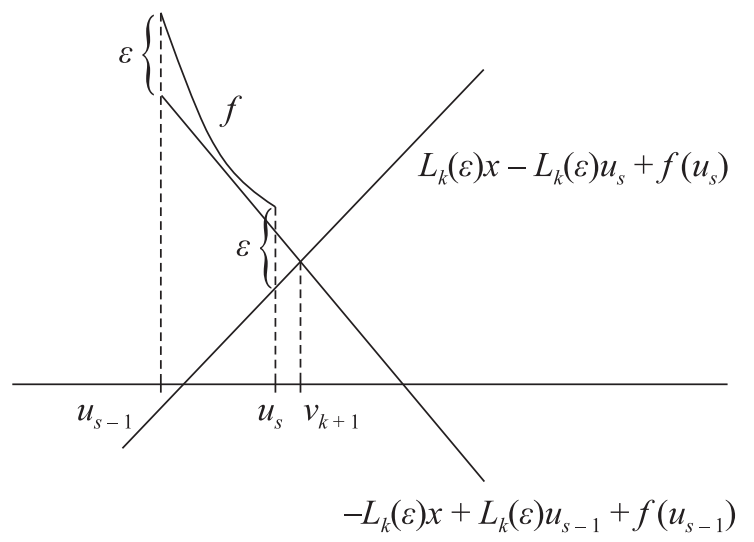

Figure 3. A situation similar to that considered in Fig. 2 when the search point $v_{k+1}$ satisfies the condition $v_{k+1}>u_{s}$

REMARK 1. It is important to note that, as distinct from the case of the Lipschitz functions, the fulfillment of condition (7) is not guaranteed for every step of the algorithm and it is explained in Figs. 1-3. If the condition fails, the algorithm can get into an infinite loop, which can take place during the numerical experiments. To avoid it we involve steps 3 and 4 . One may note that step 4 can be done by a finite number of iterations because for a fixed $u_{s-1}$ and $u_{s}$ we have $\lim _{L^{\prime}(\varepsilon) \rightarrow \infty} v_{k}=\frac{u_{s}+u_{s-1}}{2}$.

The working sequence $\left\{L_{k}(\varepsilon)\right\}$ produced during algorithm $\mathrm{A}$ is nondecreasing, starting with some number $k_{0}$ such that $l_{k_{0}}(\varepsilon)>0$ and $L_{k}(\varepsilon)>0$ for every $k \geq k_{0}$. If this number does not exist, then $L_{k}(\varepsilon)=\nu>0$ for every number $k$. 
From the construction of $L_{k}(\varepsilon), l_{k}(\varepsilon)$ and the definition of $l(\varepsilon)$ we have the following inequalities:

$$
0<L_{k}(\varepsilon) \leq \max \left\{\nu ; l(\varepsilon) \sup _{k \geq 1} \mu_{k}\right\}<\infty .
$$

Now we formulate and prove Propositions 1 and 2, which extend in a certain way Theorems 1 and 3 in [Vasil'ev, 1988, p. 53-58] to a function which is continuous on a compact interval and is not necessarily Lipschitz.

Proposal 1. Let a function $f$ be continuous on a compact interval $[a ; b]$ and the value $\varepsilon>0$ and the function $f$ be conformed on $[a ; b]$. Let the sequence $\left\{v_{k}\right\}$ constructed by the algorithm $A, v_{*}$ and $w_{*}$ be any limit points of the sequence $\left\{v_{k}\right\}$. Then the following estimate is correct:

$$
f\left(v_{*}\right)-\frac{\varepsilon}{4} \leq f\left(v_{i}\right), \quad i=0,1, \ldots
$$

and the following inequality holds

$$
\left|f\left(v_{*}\right)-f\left(w_{*}\right)\right| \leq \frac{\varepsilon}{4} .
$$

Proof. The proof is based on the ideas from Theorem 1 in [Vasil'ev, 1988, p. 53-58]. Let us introduce the following notation:

$\left[u_{m(k)-1} ; u_{m(k)}\right]$ is a sequence of intervals, each has point $v_{*}$ and an infinite number of points $v_{k}$ not the same as $v_{*}$ and, moreover, $u_{m(k)-1} \leq u_{m(k+1)-1}<u_{m(k+1)} \leq u_{m(k)}(k=1,2, \ldots)$;

$N\left(v_{*}\right)$ is the set of numbers $k \geq 1$ such that $u_{m(k)-1}<u_{m(k+1)-1}$ or $u_{m(k+1)}<u_{m(k)}$.

Repeating the proof of Theorem 1 in [Vasil'ev, 1988, p. 53-58] because of (5), we have

$$
R_{k}(m(k), \varepsilon) \geq R_{k}(i, \varepsilon), \quad i=1, \ldots, k, \quad k \in N\left(v_{*}\right) .
$$

Then,as in [Vasil'ev, 1988, p. 53-58] and taking into account that condition (7) holds for every point $v_{k}$, we have

$$
0<u_{m(k+1)}-u_{m(k+1)-1}<u_{m(k)}-u_{m(k)-1}, \quad k \in N\left(v_{*}\right),
$$

and

$$
\lim _{k \rightarrow \infty}\left(u_{m(k)}-u_{m(k)-1}\right)=0, \quad \lim _{k \rightarrow \infty} u_{m(k)-1}=\lim _{k \rightarrow \infty} u_{m(k)}=v_{*} .
$$

One can easily show that

$$
\lim _{k \rightarrow \infty} R_{k}(m(k), \varepsilon)=-4 f\left(v_{*}\right) .
$$

Indeed, if we put in (4) $i=m(k)$ and find the limit (4) increasing $k \rightarrow \infty\left(k \in N\left(v_{*}\right)\right)$ inclusive of (7), (8), (12) and because of the uniform continuity of $f$, we obtain (13).

Since we can rewrite the formula (4) as

$$
R_{k}(i, \varepsilon)=\left(L_{k}(\varepsilon)\left(u_{i}-u_{i-1}\right)+\varepsilon\right)\left(1+\frac{f\left(u_{i}\right)-f\left(u_{i-1}\right)}{L_{k}(\varepsilon)\left(u_{i}-u_{i-1}\right)+\varepsilon}\right)^{2}-4 f\left(u_{i}\right)-\varepsilon
$$

and

$$
R_{k}(i, \varepsilon)=\left(L_{k}(\varepsilon)\left(u_{i}-u_{i-1}\right)+\varepsilon\right)\left(1-\frac{f\left(u_{i}\right)-f\left(u_{i-1}\right)}{L_{k}(\varepsilon)\left(u_{i}-u_{i-1}\right)+\varepsilon}\right)^{2}-4 f\left(u_{i-1}\right)-\varepsilon,
$$

we have the following inequalities, as in the proof of Theorem 1 in [Vasil'ev, 1988, p. 53-58]:

$$
-4 f\left(u_{i}\right)+\varepsilon<R_{k}(i, \varepsilon) \leq R_{k}(m(k), \varepsilon)
$$

for every $k \in N\left(v_{*}\right)$ and $i=0,1, \ldots, k$. From this, as in [Vasil'ev, 1988, p. 53-58], one can show that

$$
f\left(v_{*}\right)-\frac{\varepsilon}{4} \leq f\left(v_{i}\right), \quad i=0,1, \ldots .
$$

Continuing the proof of Theorem 1 in [Vasil'ev, 1988, p. 53-58], we have inequality (10). 
The following set $U_{*}=\left\{u: u \in[a ; b], f(u)=f_{*}=\min _{x \in[a ; b]} f(x)\right\}$ is introduced in [Vasil'ev, 1988, p. 53-58].

Proposal 2. Let a function $f$ be continuous on a compact interval $[a ; b]$. In addition, let the following constraint hold in the algorithm A:

$$
L_{k}(\varepsilon) \geq 2 l(\varepsilon),
$$

starting with some iteration and $v_{*}$ - any limit point of the sequence $\left\{v_{k}\right\}$ constructed by the algorithm A. Then the following estimate is correct:

$$
f\left(v_{*}\right)-f_{*} \leq \varepsilon .
$$

Proof. Repeating the proof of Theorem 3 in [Vasil'ev, 1988, p. 53-58] for some point $u_{*} \in U_{*}$ $\left(u_{r(k)-1} \leq u_{*} \leq u_{r(k)}, k=1,2, \ldots\right)$ because of the condition $(1)(l(\varepsilon) \in\{L(\varepsilon)\})$ and the definition of the point $u_{*} \in U_{*}$ we have

$$
\begin{aligned}
f\left(u_{r(k)}\right)-f\left(u_{*}\right) & \leq l(\varepsilon)\left(u_{r(k)}-u_{*}\right)+\varepsilon, \\
f\left(u_{r(k)-1}\right)-f\left(u_{*}\right) & \leq l(\varepsilon)\left(u_{*}-u_{r(k)-1}\right)+\varepsilon .
\end{aligned}
$$

Summing the inequalities above, we obtain

$$
f\left(u_{r(k)}\right)+f\left(u_{r(k)-1}\right) \leq l(\varepsilon)\left(u_{r(k)}-u_{r(k)-1}\right)+2 f\left(u_{*}\right)+2 \varepsilon .
$$

By virtue of the formula (4) and condition (14) we have

$$
\begin{aligned}
R_{k}(r(k), \varepsilon) & \geq L_{k}(\varepsilon)\left(u_{r(k)}-u_{r(k)-1}\right)-2\left(f\left(u_{r(k)}\right)+f\left(u_{r(k)-1}\right)\right) \geq \\
& \geq L_{k}(\varepsilon)\left(u_{r(k)}-u_{r(k)-1}\right)-2 l(\varepsilon)\left(u_{r(k)}-u_{r(k)-1}\right)-4 f\left(u_{*}\right)-4 \varepsilon= \\
& =\left(L_{k}(\varepsilon)-2 l(\varepsilon)\right)\left(u_{r(k)}-u_{r(k)-1}\right)-4 f\left(u_{*}\right)-4 \varepsilon \geq-4 f\left(u_{*}\right)-4 \varepsilon .
\end{aligned}
$$

Hence,

$$
R_{k}(r(k), \varepsilon) \geq-4 f\left(u_{*}\right)-4 \varepsilon, \quad k=1,2, \ldots
$$

From (15), taking $i=r(k)$ and (16) as in [Vasil'ev, 1988, p. 53-58], we have the following inequalities:

$$
R_{k}(m(k)) \geq R_{k}(r(k)) \geq-4 f\left(u_{*}\right)-4 \varepsilon \geq-4 f\left(v_{*}\right)-4 \varepsilon .\left(k \in N\left(v_{*}\right)\right) .
$$

Taking condition (13) into account, we have

$$
\lim _{k \rightarrow \infty} R_{k}(m(k))=-4 f\left(v_{*}\right) \geq-4 f\left(u_{*}\right)-4 \varepsilon \geq-4 f\left(v_{*}\right)-4 \varepsilon
$$

and from this we directly derive the estimate (15).

REMARK 2. As in [Strongin, 1978; Vasil'ev, 1988], one can show that condition (14) can be guaranteed by an appropriate selection of the method parameters $\nu$ and $\mu_{k}$ :

$$
\nu \geq 2 l(\varepsilon), \quad \mu_{k} \geq 2 \frac{l(\varepsilon)}{l_{k}(\varepsilon)} .
$$

It is clear that if the values of $l(\varepsilon)$ and $l_{k}(\varepsilon)$ are unknown, then the appropriate selection of $\nu$ and $\mu_{k}$ can be performed by repeating the algorithm with various method parameters. The work [Zabotin, Chernyshevskij, 2018] can be useful in deriving the estimate of $l(\varepsilon)$.

In conclusion, we present some results of the numerical experiments performed by the developed algorithm. 
EXAMPLE 1. In [Arutyunova, 2013] the minimum of a function

$$
f_{1}(x)=\min \{\sqrt{|x+4|}-1 ; \sqrt{|x+1|}-1.005 ; \sqrt{|x-3|}+0.5\}
$$

was found on the intervals $[-5 ; 5]$ and $[-10 ; 10]$ by the modified Yevtushenko method with the previously obtained dependence of the constant $l(\varepsilon)$ on the value of $\varepsilon$. By using the proposed algorithm $\mathrm{A}$ for the specified cases, we found the minimum point and the minimal function value without knowledge of any a priori estimate of $l(\varepsilon)$. The computational results are presented in Table 1 . The following values of the method parameters were used: $v=1, \mu_{0}=2, \mu_{k}=2(k=1,2, \ldots)$. For a given accuracy $\delta>0$ we chose the following termination condition:

$$
\left\{\begin{array}{l}
\left|f\left(x_{k+1}\right)-f\left(x_{k}\right)\right| \leq \delta \\
\left|x_{k+1}-x_{k}\right| \leq \delta
\end{array}\right.
$$

Computational results agree exactly with the results in [Arutyunova, 2013]. For clarification, a function graph is shown in Fig. 4.

Table 1. The results of minimization of the function $f_{1}(x)=\min \{\sqrt{|x+4|}-1 ; \sqrt{|x+1|}-1.005 ; \sqrt{|x-3|}+0.5\}$ by the proposed algorithm. The following notation is used: $x_{*}$ is the minimum point derived by the algorithm, $f_{*}$ is the approximate minimum value, $L_{n}(\varepsilon)$ is the approximate estimate of the $l(\varepsilon)$ constant, $\delta>0$ and $\varepsilon>0$

\begin{tabular}{|c|c|c|c|c|c|c|}
\hline Interval & Accuracy $\delta>0$ & $\varepsilon$ & $x_{*}$ & $f_{*}$ & Number of steps & $L_{n}(\varepsilon)$ \\
\hline \multirow{6}{*}[-5;5]{} & \multirow{2}{*}{0.01} & 0.005 & -4.002216430 & -0.952921022 & 97 & 24.39441821 \\
\hline & & 0.001 & -4.284787036 & -0.466345583 & 1058 & 388.34939613 \\
\hline & \multirow{2}{*}{0.001} & 0.005 & -1.000002183 & -1.003522488 & 309 & 69.2886888 \\
\hline & & 0.001 & -0.999998643 & -1.003835130 & 1534 & 388.34939613 \\
\hline & \multirow{2}{*}{0.0001} & 0.005 & -1.000002183 & -1.003522488 & 309 & 69.2886888 \\
\hline & & 0.001 & -0.999999993 & -1.00491671 & 1608 & 413.02619174 \\
\hline \multirow{6}{*}[-10;10]{} & \multirow{2}{*}{0.01} & 0.005 & -0.999999692 & -1.004445223 & 379 & 60.134909 \\
\hline & & 0.001 & -3.999518109 & -0.978048002 & 232 & 38.042857 \\
\hline & \multirow{2}{*}{0.001} & 0.005 & -0.999999696 & -1.004448987 & 498 & 88.904087 \\
\hline & & 0.001 & -0.999998028 & -1.003596067 & 2057 & 352.695801 \\
\hline & \multirow{2}{*}{0.0001} & 0.005 & -0.999999696 & -1.004448987 & 498 & 88.904087 \\
\hline & & 0.001 & -0.999999938 & -1.004751327 & 2131 & 363.081258 \\
\hline
\end{tabular}
are method parameters

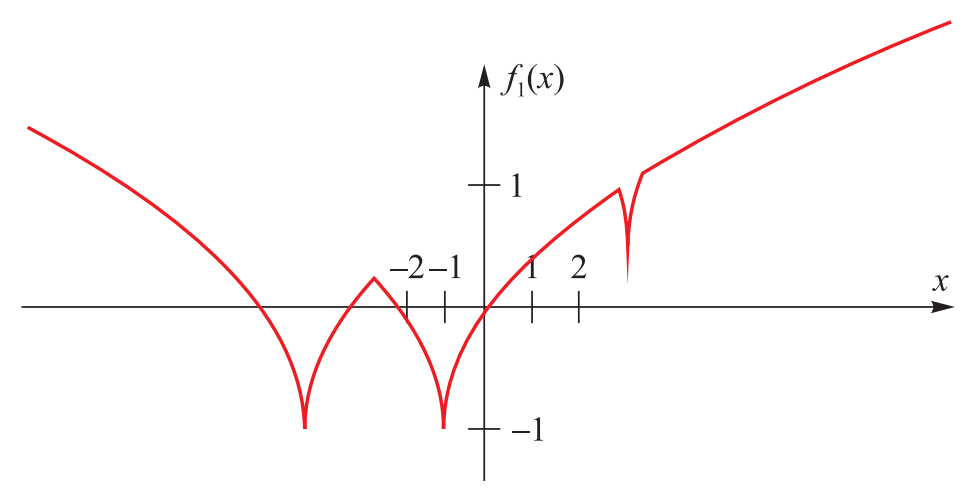

Figure 4. A graph of the function $f_{1}(x)$ whose global minimum was found by the proposed algorithm 
EXAMPLE 2. We find a minimum for the function

$$
f_{2}(x)=\left\{\begin{array}{l}
-\arcsin (x+2), \quad-3 \leq x \leq-1, \\
\arcsin x, \quad-1 \leq x \leq 0, \\
-\arcsin x, \quad 0 \leq x \leq 0.9,
\end{array}\right.
$$

on the interval $[-3 ; 0.9]$. In [Arutyunova et al., 2017] for the function $\arcsin x$ the $l(\varepsilon)$ estimate was derived. It should be noted again that the results given below do not require knowledge of an a priori $l(\varepsilon)$ estimate. The computational results are presented in Table 2. For clarification, a graph of the function considered is shown in Fig. 5.

Table 2. Results of minimization of the function $f_{2}(x)$ by the proposed algorithm. The following notation is used: $x_{*}$ is the minimum point derived by the algorithm, $f_{*}$ is the approximate minimum value, $L_{n}(\varepsilon)$ is the approximate estimate of the $l(\varepsilon)$ constant, and $\delta>0$ and $\varepsilon>0$ are the method parameters

\begin{tabular}{|c|c|c|c|c|c|c|}
\hline Interval & Accuracy & $\varepsilon$ & $x_{*}$ & $f_{*}$ & Number of steps & $L_{n}(\varepsilon)$ \\
\hline \multirow{5}{*}[-3;0.9]{} & \multirow{2}{*}{0.01} & 0.005 & -0.999999322 & -1.569632160 & 158 & 193.415676 \\
\cline { 3 - 7 } & \multirow{2}{*}{0.001} & 0.001 & -0.967354969 & -1.314576854 & 384 & 485.179488 \\
\cline { 3 - 7 } & \multirow{2}{*}{0.0001} & 0.001 & -0.999999849 & -1.570247277 & 159 & 193.415676 \\
\cline { 3 - 7 } & & 0.005 & -0.999999997 & -1.5707287854 & 697 & 854.145954 \\
\cline { 3 - 7 } & & 0.001 & -0.999999999 & -1.570761253 & 162 & 193.415676 \\
\hline
\end{tabular}

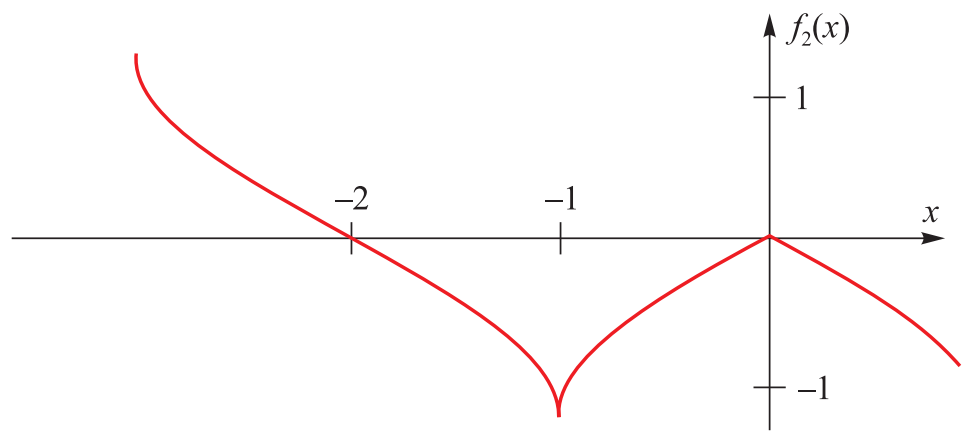

Figure 5. A graph of the function $f_{2}(x)$ whose global minimum was found by the proposed algorithm

\section{References}

Arutyunova N.K. Metod Yevtushenko poiska global'nogo minimuma $\varepsilon$-lipshitsevoy funktsii i yego prilozheniya [Yevtushenko's method for finding $\varepsilon$-Lipschitzian function global minimum and its application] // Bulletin of the KSTU-KAI Named After A. N. Tupolev. - 2013. - Vol. 2, Issue 2. P. 154-157 (in Russian).

Arutyunova N.K., Dulliev A.M., Zabotin V.I. Models and methods for three external ballistics inverse problems // Bulletin of the South Ural State University: Series "Mathematical modelling, Programming \& Computer software". - 2017. - Vol. 10, No. 4. - P. 78-91.

Arutyunova N. K., Dulliev A. M., Zabotin V.I. Algorithm for point projection on continuous on compact domain function level surface // Comput. Math. Math. Phys. - 2014. - Vol. 54, No. 9. - P. 1448 1454. 
Chernyaev Yu.A. Convergence of the gradient projection method and Newton's method as applied to optimization problems constrained by intersection of a spherical surface and a convex closed set // Comput. Math. Math. Phys. - 2016. - Vol. 56, No. 10. - P. 1716-1731.

Chernyaev Yu.A. An extension of the gradient projection metod and Newton's method to extremum problems constrained by a smooth surface // Comput. Math. Math. Phys. - 2015. - Vol. 55, No. 9. - P. 1451-1460.

Gelbaum B.R., Olmsted J.M.H. Counterexamples in analysis // New York: Dover Publications, Inc., Mineola, 2003. - 194 p.

Piyavskij S. A. Odin algoritm otyskaniya absolyutnogo minimuma funktsiy [An algorithm for finding the absolute minimum of a function] // Theory of Optimal Solutions, IK Akad. Nauk USSR, Kiev. - 1967. - Vol. 2. - P. 13-24 (in Russian).

Piyavskij S. A. A certain algorithm for seeking the absolute extremum of a function // USSR Comput. Math. Math. Phys. -1972. - Vol. 12, Issue 4. - P. 57-67.

Sergeyev Ya.D., Kvasov D.E. Diagonal'nyye metody global'noy optimizatsii [Diagonal global optimization methods]. - Moscow: Fizmatlit Publ., 2008. - 352 p. (in Russian).

Shubert B. A sequential method seeking the global maximum of a function // SIAM J. Numer. Anal. 1972. - Vol. 9. - P. 379-388.

Strongin R.G. Chislennye metody $\mathrm{v}$ mnogojekstremal'nyh zadachah [Numerical methods in multiextremal problems]. - Moscow: Nauka Publ., 1978. - 240 p. (in Russian).

Vanderbei R. J. Extension of Piyavskii's Algorithm to Continuous Global Optimization // Journal of Global Optimization. - 1999. - Vol. 14. - P. 205-216.

Vasil'ev F.P. Chislennye metody reshenija jekstremal'nyh zadach: Ucheb. posobie dlja vuzov [Numerical methods for solving extremum problems]. - Moscow: Nauka Publ., 1988. - 552 p.

Yevtushenko Yu. G. Numerical methods for finding global extrema (Case of a non-uniform mesh) // USSR Comput. Math. Math. Phys. - 1971. - Vol. 11, Issue 6. - P. 34-54.

Zabotin V.I., Arutyunova N.K. Two algorithms for finding point projection on nonconvex set in normalized space // Comput. Math. Math. Phys. - 2013. - Vol. 53, No. 3. - P. 344-349.

Zabotin V.I., Chernyaev Yu. A. A generalization of the gradient projection method to extremum value problems with preconvex constraints // Comput. Math. Math. Phys. - 2001. - Vol. 41, No. 3. P. 340-346.

Zabotin V.I., Chernyshevskij P. A. Algoritm vychisleniya minimal'noy otsenki $\varepsilon$-postoyannoy Lipshitsa nepreryvnoy funktsii [An algorithm for calculation the minimal estimation of the Lipschitz $\varepsilon$ constant of a continuous function] // Bulletin of the KSTU-KAI Named After A.N. Tupolev. 2018. - Vol. 2, Issue 2. - P. 127-132 (in Russian). 